Itinéraires Itinéraires

Littérature, textes, cultures

2009-3 | 2009

Modernités occidentales et extra-occidentales

\title{
Antoine Compagnon, Les Cinq paradoxes de la modernité
}

Émilie Sevrain

\section{OpenEdition}

\section{Journals}

Édition électronique

URL : http://journals.openedition.org/itineraires/547

DOI : 10.4000/itineraires.547

ISSN : 2427-920X

Éditeur

Pléiade

\section{Édition imprimée}

Date de publication : 1 novembre 2009

Pagination : 171-173

ISBN : 978-2-296-10115-9

ISSN : 2100-1340

\section{Référence électronique}

Émilie Sevrain, « Antoine Compagnon, Les Cinq paradoxes de la modernité », Itinéraires [En ligne], 2009-3 | 2009, mis en ligne le 26 juin 2014, consulté le 10 décembre 2020. URL : http:// journals.openedition.org/itineraires/547 ; DOI : https://doi.org/10.4000/itineraires.547

\section{cc) (1)}

Itinéraires est mis à disposition selon les termes de la licence Creative Commons Attribution - Pas d'Utilisation Commerciale - Pas de Modification 4.0 International. 


\section{Comptes rendus}

Les comptes rendus qui suivent portent essentiellement sur des ouvrages récents qui problématisent la question de la (des) modernité(s). Nous avons néanmoins inclus deux ou trois ouvrages plus anciens qui sont des références sur la question.

Antoine Compagnon, Les Cinq paradoxes de la modernité, Paris, Seuil, 1990, $190 \mathrm{p}$.

Les concepts de «moderne », «modernité » et «modernisme » ont été l'objet de nombreux travaux critiques dans les domaines français et anglo-saxons au cours de ces dernières décennies. Les définitions et lectures, parfois convergentes ou conflictuelles, de ces catégories dévoilent les ambiguités théoriques qui ont traversé l'histoire récente des beaux-arts. Dans Les Cinq paradoxes de la modernité, Antoine Compagnon propose un panorama critique des divers processus de modélisation artistique et enjeux idéologiques qui ont sous-tendu l'esthétique moderne. Selon une perspective comparative, l'auteur met en lumière, par la chronique des innovations littéraires et picturales d'illustres auteurs, les moments de crises majeures des traditions culturelles et imaginaires nationaux de nos sociétés contemporaines.

La première partie de cet essai retrace, au travers de l'ambivalence des premiers modernes, en particulier Baudelaire et Manet, le prestige du nouveau comme valeur ultime dans l'ordre du goût et de la connaissance. Sous l'influence de la révolution sociale et industrielle du XIX ${ }^{\mathrm{e}}$ siècle, la littérature et l'art remirent en cause les fondements formels et éthiques classiques. Cette négation des modèles établis offrit alors la possibilité et la revendication d'une esthétique du changement. Mais la notion de modernité, dont l'avènement est communément daté en France par la publication de l'ouvrage de Baudelaire sur «Le Peintre de la vie moderne », fut d'emblée placée sous le signe de l'équivoque. Si elle fut définie comme perception et représentation du présent, «assentiment à l'immédiateté », la modernité, prise dans le cycle continuel du temps, fut sans cesse encline à la décadence et au renouvellement. D'après l'interprétation baudelairienne de l'œuvre picturale de Guys, Compagnon distingue 
quelques traits caractéristiques de la tradition moderne, comme le non-fini, le fragmentaire, l'insignifiance et l'autonomie. « Telle [fut] la condition d'une modernité qui ne reconnait plus d'extériorité par rapport à son art, plus de codes ni de sujet, et qui doit donc se donner elle-même ses règles, modèles et critères » (p. 36). Par l'ébranlement des procédés artistiques et l'insolence des transpositions picturales, Le Déjeuner sur l'herbe de Manet représenta l'un des exemples privilégiés de l'œuvre moderne.

Si nombre des créations de Courbet et Manet, comme celles de Flaubert et Baudelaire firent scandale, aucun d'eux ne perçut le nouveau comme une contribution au futur; bien au contraire, ils recherchèrent dans l'art l'essence du présent «en sa qualité de présent», sans juger leur pratique artistique comme esthétique avant-gardiste. Comme l'écrit Compagnon, « on confond pourtant trop souvent modernité et avant-garde » (p. 48). Or, le principal critère de distinction entre ces deux notions se fonde autour de la valeur du futur comme conscience du temps. Les néo-impressionnistes furent les premiers à nourrir le sentiment d'être historiquement en avance sur leurs contemporains. Si certains peintres s'engagèrent dans un projet de réforme esthétique, d'autres, tels que Seurat ou Signac, utilisèrent leur art au service d'une révolution politique. Le glissement sémantique de l'avant-garde, comme «dogme du progrès » puis « futuriste en soi », correspondit à l'évolution de la critique formaliste qui vit en l'innovation artistique le principe même de l'explication théorique. Vers la fin du XIX $X^{\mathrm{e}}$ siècle, nombre de récits orthodoxes tentèrent de définir, avec une intention à la fois apologétique et théologique, les tendances contradictoires de l'avant-garde. Que les modèles soient choisis dans les domaines poétique ou pictural, le présupposé historico-génétique des théoriciens, en particulier Greenberg ou Friedrich, fut réduit à une dialectique de la purification de plus en plus radicale de la tradition artistique. «L'histoire de l'art moderne se racon[ta] ainsi comme la quête du degré zéro et de la pureté absolue » (p. 68), excluant paradoxalement les composantes essentielles de la modernité, telles que l'ironie ou la mélancolie.

Les prétentions théoriciennes développées dans nombre de manifestes avant-gardistes furent souvent peu conformes aux pratiques artistiques des premiers modernes. Cependant, comme le souligne Compagnon, « la distinction du contenu et de la forme, signalée par les esthétiques du XVIII ${ }^{\mathrm{e}}$ siècle, puis l'autonomie croissante de la forme au cours du XIX ${ }^{\mathrm{e}}$ siècle, furent sans doute parmi les conditions qui rendirent l'abstraction possible » (p. 88). Par le renoncement à l'objet et la recherche de lignes épurées, les artistes tentèrent d'exprimer les vérités spirituelles d'un monde invisible. De manière paradoxale, les fondateurs de l'art abstrait, Kandinsky, Mondrian et Malevitch, recoururent à des doctrines surannées, en particulier le spiritualisme, la théosophie ou le nihilisme, afin de légitimer leurs pratiques nouvelles et intuitives. À l'inverse, les déclarations théoriciennes du surréalisme, radicales et polémiques, suscitèrent un nouvel académisme. Si les créations surréalistes détenaient leur force suggestive de la déformation délirante du monde réel ou de la juxtaposition arbitraire d'objets ordinaires, la spontanéité et l'automatisme psychique ne régirent progressivement plus certaines productions artistiques. «De la même façon que la poésie surréaliste aboutit 
rapidement à un certain nombre de poncifs, la peinture onirique de Chirico, Dali et Magritte, rencontra vite ses lieux communs et fournit les vignettes de la société de consommation » (p. 107).

Dans le chapitre intitulé « Le marché des dupes : expressionnisme et pop art», Compagnon nous propose de réfléchir sur les procédés de médiatisation et de disqualification de l'art moderne. Durant la Seconde Guerre mondiale, de nombreux collectionneurs et artistes surréalistes s'exilèrent en Amérique pour fuir la montée du fascisme et du nazisme en Europe. Face à la dévastation des systèmes politiques et économiques de l'hexagone, les marchés américains acquirent une position dominante. Sous l'impulsion de Pollock, Newman ou encore Motherwell, l'expressionnisme abstrait, caractérisé par « une coïncidence parfaite de l'émotion, du geste et du tracé » (p. 122), apparut comme l'ultime avatar de la culture d'élite. En réaction à l'institution artistique, le pop art, « art conceptuel promu art de masse » (p. 124), amorça un mouvement de désacralisation du nouveau dans la société de consommation. Les ready-made de Duchamp ou les collages photographiques de Warhol affectèrent les critères esthétiques du beau et de l'unicité, symboles du goût bourgeois. L'œuvre, dépourvue de transcendance, devint une marchandise, un objet de spéculation des marchés médiatiques et industriels. Emblème paradoxal d'une révolution culturelle pour les générations européennes de 1968, le pop art affranchit l'art du dogme de l'innovation, fondement élitiste et ésotérique de la tradition moderne.

Dans ce contexte d'optimisme technologique et de valorisation de la culture populaire, apparut le terme de « postmodernisme ». En opposition à la conception standardisée de l'urbanisme d'après-guerre, le courant postmoderne, réservé initialement au domaine architectural, revendiqua le droit à l'éclectisme, au retour des motifs du passé et au mélange des influences étrangères, comme le baroque et le maniérisme. Par son triomphe international, cette nouvelle esthétique eut une influence certaine sur l'ensemble des beaux-arts. En peinture, réagissant contre le « darwinisme linguistique » et « l'évolutionnisme culturel », la subjectivité de l'artiste et le contentement du spectateur sont exaltés. En littérature, la prose romanesque, dépourvue des conventions élitistes du réalisme et du purisme, présenta de nouvelles caractéristiques formelles, notamment « l'indétermination du sens », « la mise en cause de la narration », « la rétraction de l'auteur» ou encore « l'interpellation du lecteur ». Mais, en cette période de crise généralisée des idéaux culturels modernes, des divergences théoriques apparurent, actualisant la problématique persistante des rapports entre tradition et innovation, imitation et originalité.

Par l'analyse de nombreuses œuvres picturales, plastiques et poétiques - que l'on aurait sans doute souhaité élargir ponctuellement au domaine musical français et anglo-saxon, cet ouvrage met en lumière les subversions et apories majeures de la tradition moderne, favorisant une meilleure compréhension des renouvellements formels et des effets de réception contradictoires de l'histoire récente des beaux-arts. 